The internal kinematics of dwarf spheroidal galaxies

G. Mamon, F. Combes, C. Deffayet, B. Fort (eds)

EAS Publications Series, Vol. ?, 2005

\title{
THE INTERNAL KINEMATICS OF DWARF SPHEROIDAL GALAXIES
}

\author{
Mark I. Wilkinson ${ }^{1}$, Jan T. Kleyna ${ }^{2}$, N. Wyn Evans ${ }^{1}$, Gerard F. \\ Gilmore $^{1}$, Justin I. Read ${ }^{1}$, Andreas Koch ${ }^{3}$, Eva K. Grebel ${ }^{3}$ and Michael \\ J. Irwin ${ }^{1}$
}

\begin{abstract}
The status of kinematic observations in Local Group dwarf spheroidal galaxies (dSphs) is reviewed. Various approaches to the dynamical modelling of these data are discussed and some general features of dSph dark matter haloes based on simple mass models are presented.
\end{abstract}

\section{Introduction}

The dwarf spheroidal galaxies (dSph) of the Local Group constitute a valuable testing ground for theories of dark matter and galaxy formation. The internal kinematics of dSphs suggest that they are dark matter dominated at all radii, making them the smallest stellar systems known to contain dynamically significant quantities of dark matter. Cosmological simulations of galaxy formation predict the existence of many sub-haloes in the halo of a Milky Way type galaxy - however, it is currently unclear which of the objects in the simulations correspond to the observed dSphs. A determination of the kinematic properties of dSphs, and in particular of their masses and mass distributions, is an important step towards understanding both their origin in a cosmological context and their subsequent evolution within the Local Group.

\section{Kinematic Observations in dSphs}

The field of dSph kinematics had its origins in a seminal paper by Aaronson et al. (1983) which estimated the velocity dispersion of Draco using velocity measurements of three carbon stars. In that paper, a mass to light ratio of 30 was

\footnotetext{
${ }^{1}$ Institute of Astronomy, Madingley Road, Cambridge CB3 0HA, UK

2 Institute for Astronomy, 2680 Woodlawn Drive, Honolulu, Hawaii 96822-1897, USA

3 Astronomical Institute of the University of Basel, Venusstrasse 7,CH 4102 Binningen, Switzerland
}

(c) EDP Sciences 2018

DOI: (will be inserted later) 
The internal kinematics of dwarf spheroidal galaxies

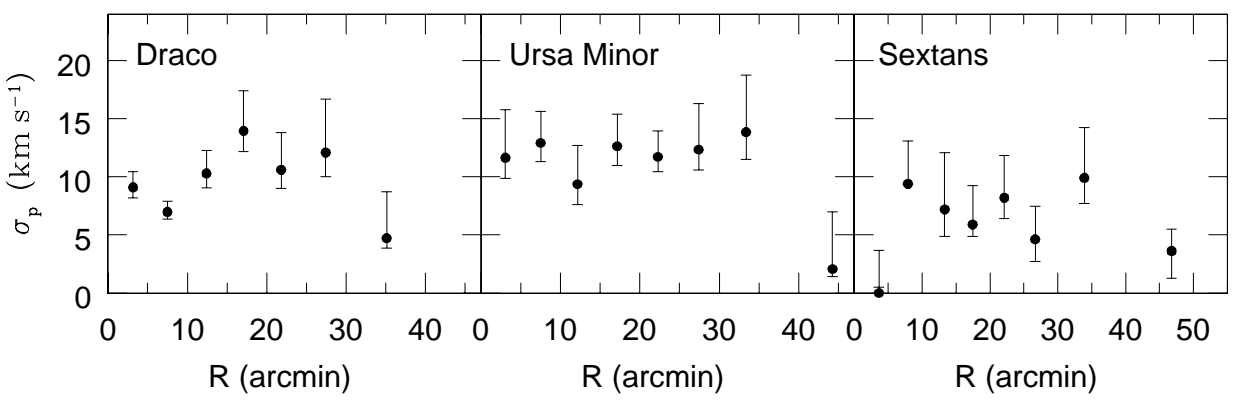

Fig. 1. Velocity dispersion profiles for the Draco, Ursa Minor and Sextans dSphs (Kleyna et al. 2004; Wilkinson et al. 2004).

tentatively inferred from the measured dispersion of $6.5 \mathrm{~km} \mathrm{~s}^{-1}$. This provided the first hint that the dSphs were a class of stellar system distinct from the globular clusters. By 1987, the number of stars with measured velocities in Draco had been increased to eleven, and an additional ten stars had been observed in Ursa Minor (Aaronson \& Olszewski 1987). Based on the associated estimates of the velocity dispersions of both galaxies, Pryor \& Kormendy (1990) used a family of two-component (luminous plus dark matter) King models to demonstrate that the central dark matter density in these dSphs must be greater than $0.05 \mathrm{M}_{\odot} \mathrm{pc}^{-3}$. They also showed that extended dark matter haloes in dSphs could be compatible with both the kinematics and stellar density distributions.

Throughout the 1990s, the number of dSphs with measured velocity dispersions increased steadily - Mateo (1998) cites dispersion values for ten systems. However, dSph velocity distributions were still represented by a single number, the value of the central velocity dispersion. Mateo (1997) published the first velocity dispersion profile for a dSph, showing the variation of the velocity dispersion as a function of projected radius in Fornax. The measured profile, based on 215 individual velocities, showed that the dispersion of Fornax remains approximately flat almost to the edge of the light distribution. This profile is inconsistent with simple models of Fornax with a constant mass to light ratio and an isotropic velocity distribution.

The availability of multi-fibre and multi-slit spectrographs on $4 \mathrm{~m}$ and $10 \mathrm{~m}$ class telescopes has revolutionised the study of dSph kinematics by providing an efficient means to obtain large data sets of stars in each target dSph. Kleyna et al. (2001) presented the dispersion profile of Draco based on velocities for 159 stars obtained using the WYFFOS multi-fibre spectrograph on the William Herschel Telescope, La Palma. Subsequently, the measurement of dispersion profiles has continued apace using instruments on all the major telescopes: WYFFOS (WHT, La Palma), FLAMES/GIRAFFE \& UVES (VLT), GMOS (Gemini), MIKE (Magellan), DEIMOS and HIRES (Keck) and dispersion profiles are now available (or will appear shortly) for almost all the Local Group dSphs (e.g. Kleyna et al. 2004; Tolstoy et al. 2004; Wilkinson et al. 2004; Muñoz et al. 2005; Wang et al. 2005). Figure 1 1 shows the profiles of Draco, Ursa Minor and Sextans based on the WHT 


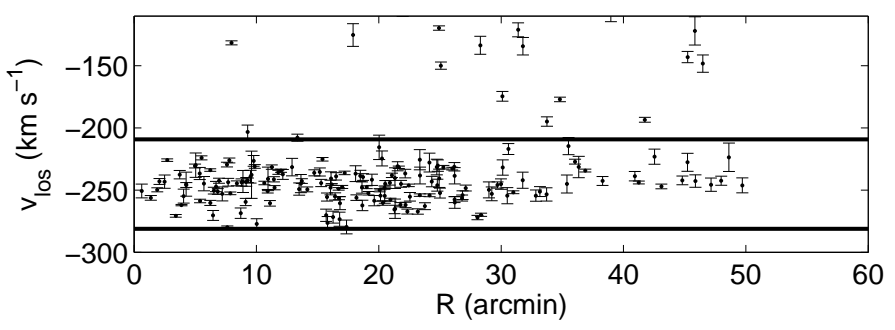

Fig. 2. Line of sight velocity versus projected radius for the Ursa Minor data set of Wilkinson et al. (2004), including some non-members. The horizontal lines indicate the $3 \sigma$ velocity cuts used to determine membership. See text for a discussion.

observations of Wilkinson et al.(2004) and Kleyna et al. (2004).

All the teams involved in this effort measure the velocities of giant branch stars in the dSphs, typically using the Ca triplet absorption lines. Velocity errors of $1-5$ $\mathrm{km} \mathrm{s}^{-1}$ are routinely obtained. In each target galaxy, the goal is to obtain a data set of several hundred velocities covering the entire face of the stellar distribution. In addition, higher resolution spectra (such as those from FLAMES at the VLT) are being used to obtain abundance estimates for large numbers of stars (e.g. 401 stars in Sculptor: Tolstoy et al. 2004; 437 stars in Carina: Koch et al. 2005).

The new instruments and larger data sets have made it possible to investigate empirically the extent to which the measured velocity dispersions might be inflated by non-dynamical effects. In particular, atmospheric "jitter" at the level of a few $\mathrm{km} \mathrm{s}^{-1}$, which is observable in AGB stars, is not a problem for the stars below the tip of the giant branch which now represent the vast majority of the kinematic samples. The orbital velocities of binary stars were also a serious concern for early samples based on small, single-epoch data sets. However, Olszewski et al. (1996) used repeated observations of a sample of 118 stars in Draco and Ursa Minor to demonstrate that the scatter in velocities due to binaries is small compared to the observed dispersions of the dSphs. This was later confirmed by Kleyna et al. (2002) based on repeat observations of 61 stars in Draco from the sample of Armandroff et al. (1995) with a baseline of 6-8 years. Thus, the large amplitudes of the observed velocity dispersions appear to be reliable.

\subsection{Cold outer populations: are they real?}

The velocity dispersion profiles of Draco and Ursa Minor shown in Figure \display the unexpected property that at large radii there is a sudden fall-off in the projected dispersion. Such a feature is difficult to understand in the context of smooth, equilibrium models of dSphs. Wilkinson et al. (2004) suggest that tidal sculpting of the outer regions of a dSph by the Milky Way could give rise to such a feature. However, Read et al. (2005) have demonstrated that tidal effects cannot produce steeply falling dispersion profiles, even in projection.

Recently, Muñoz et al. (2005) have measured velocities at very large angular 
The internal kinematics of dwarf spheroidal galaxies

distances from Ursa Minor. Based on these velocities, they conclude that the dispersion at the largest radii is approximately flat. Due to the small number of stars at large radii, however, their final dispersion point contains stars ranging in radius from $1.5-6 \mathrm{kpc}$ (corresponding to $1-4$ times the limiting radius of Ursa Minor). Velocity gradients (such as might be expected if the stars are associated with a tidal tail) could lead to an apparent increase in the measured dispersion over this radial range. It is also pertinent to consider the distribution of velocities in Ursa Minor (Figure 2). By eye, the velocity distribution appears to narrow outside 37 arcmin in this plot - 9 out of the 12 stars in this region lie within error at the mean velocity of the entire sample. Application of an F-test (e.g. Press et al. 1992) to the velocity distributions inside and outside $37^{\prime}$ shows that the outer stars have a dispersion which is 2.6 times smaller than the value for stars inside this radius (90 per cent confidence). It therefore appears that the velocity distribution between $37^{\prime}$ and $50^{\prime}$ is indeed narrower than that inside $37^{\prime}$. If it is confirmed that beyond $50^{\prime}$ the dispersion increases again, then this might be consistent with the onset of tidal effects in that region. What is clear is that the velocity distributions in the outer regions of some dSphs are significantly more complicated than was previously thought and further data are required to resolve the situation. The origin of the dispersion profile features awaits satisfactory explanation - observations are underway to increase the numbers of observed stars at the largest radii in these dSphs.

\section{Dynamical models of dSphs}

As was stated earlier, the key goal for dynamical models of dSphs is to determine the masses, extents and mass profiles of their dark matter haloes. Knowledge of these properties would make it possible to investigate, for example, the extent to which dSphs share similar halo properties despite the wide variation in the properties of their stellar components (stellar mass, number of distinct stellar populations, etc). An important question which arises in the context of mass modelling is the extent to which the dSphs have been affected by their proximity to the Milky Way or M31. It is clear that at some level the dSphs must have been perturbed by tidal forces. However, there is evidence that for at least some of the dSphs, tidal effects have not had a significant impact in the region probed by the stellar distribution (e.g. Draco: Klessen et al. 2003). The issue of tides remains controversial, but it is worth noting that so far no unambiguous kinematic signature of tidal effects in a dSph has been observed. In addition to testing models of tidal perturbation, the dSphs also provide a potential test of MOdified Newtonian Dynamics - a discussion of the performance of MOND in the case of Draco is presented by Lokas et al. (2005).

All mass modelling of dSphs to date has been based on the assumption of virial equilibrium. Aside from the question of whether this assumption is valid, there are considerable uncertainties associated with the outer surface brightness profiles of dSphs - deeper imaging often reveals breaks in the outer profiles (e.g. Carina: Majewski et al. 2005). As was discussed earlier, the detailed structure of the observed 

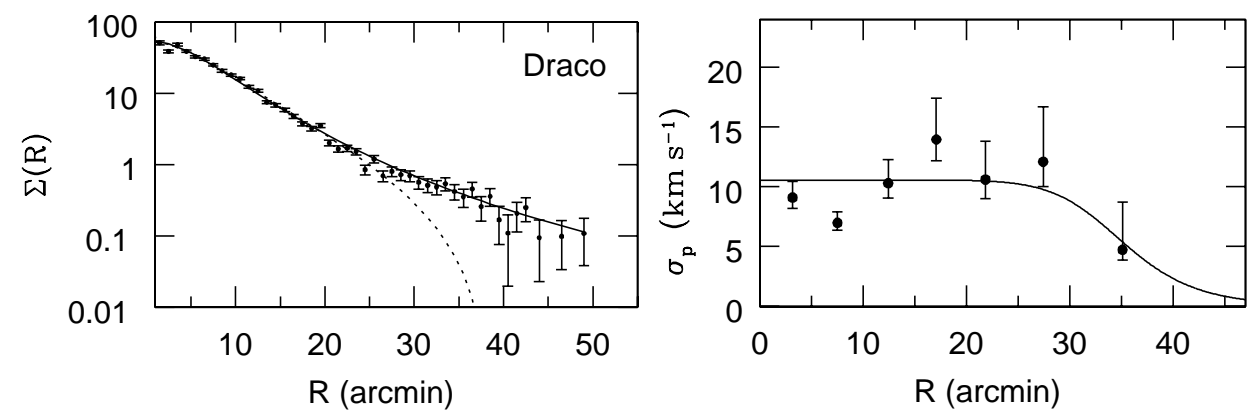

Fig. 3. Surface brightness and velocity dispersion profiles of Draco (Wilkinson et al. 2004). Solid curves show the assumed smooth profiles used in the analysis presented here.

outer velocity dispersion profiles is also controversial. The uncertainties associated with the two main observed inputs necessarily introduce large uncertainties into the resulting mass estimates which are difficult to quantify.

Notwithstanding the uncertainties in the outermost regions, a number of authors have published mass models for dSphs based on the (presumably) more robust data inside the break radii in the light distributions. Pryor \& Kormendy (1990) introduced two-component models to the study of dSphs, to explore the possibility the dSphs have extended haloes similar to those found in external galaxies. More recently, mass models based either on velocity moments (e.g. Łokas 2001 and Lokas et al. 2005) or on direct comparison of two parameter distribution functions with the observed data (e.g. Kleyna et al. 2002) have appeared in the literature. To date, Draco and Ursa Minor have received the most attention - however, Wang et al. (2005) have recently applied a non-parametric modelling scheme to new data on Fornax to derive simultaneously the velocity dispersion profile and mass profile in the context of an isotropic velocity distribution.

\section{Mass models from Jeans equations}

To investigate the general properties of dSph haloes, we will now derive some simple mass models for four Milky Way dSphs using Jeans equations. We assume smooth functions to represent the light distribution and velocity dispersion profiles of each dSph - Figure 3 shows the assumed functions for Draco. Under the simplifying assumptions of spherical symmetry and an isotropic velocity distribution, the Jeans equations give rise to the simple mass estimator (see e.g. Binney \& Tremaine 1987)

$$
M(r)=-\frac{r^{2}}{G}\left(\frac{1}{\nu} \frac{\mathrm{d} \nu \sigma_{r}^{2}}{\mathrm{~d} r}\right)
$$

where $\nu(r)$ is the three-dimensional light density distribution and $\sigma_{r}(r)$ is the radial velocity dispersion. The latter two quantities are obtained by straightforward 

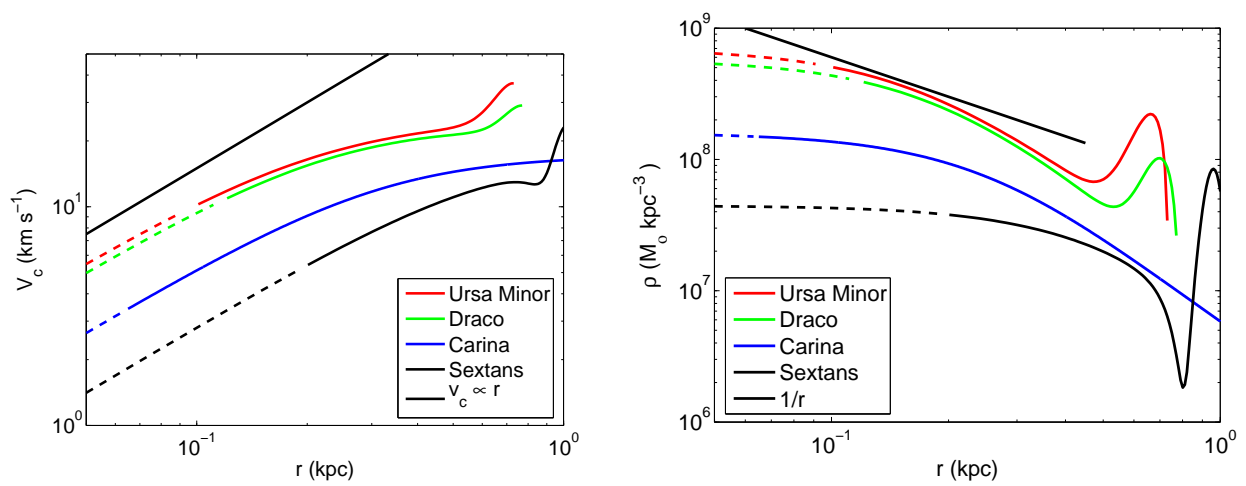

Fig. 4. Circular speed curves (left) and density profiles (right) for four Milky Way dSphs, derived using Jeans equations. From top to bottom the curves show the results for Ursa Minor, Draco, Carina and Sextans. Also shown at the top of each plot are the expected curves for an $r^{-1}$ density distribution. See text for a detailed discussion.

deprojection of the surface brightness profiles and projected velocity dispersions.

The left panel of Figure 4 shows the circular speed $v_{c}=\sqrt{G M(r) / r}$ as a function of radius obtained via equation 4.1. The curves are broken in the inner regions where either the data or the model assumptions (or both) break down - the outer regions exhibit unphysical behaviour due to the features in the light and/or velocity distributions which, as discussed above, cannot be represented by simple, smooth models. The enclosed masses (within the largest radii where the models are plausible) lie in the range $3-8 \times 10^{7} \mathrm{M}_{\odot}$. The right panel of Figure 4 shows the associated mass density distributions. Two features of these curves are noteworthy. First, all four density profiles tend towards central logarithmic slopes which are considerably shallower than the value of about -1 expected on the basis of cosmological simulations. Secondly, more massive haloes have higher central densities.

One should be cautious about over-interpreting the results of this analysis, due to the simplifying assumptions which have been made. However, on the basis of these results it is clear that the data do not drive one to assume cusped haloes for dSphs - cored haloes with isotropic stellar velocity distributions in the inner regions would appear to be broadly consistent with the current data.

It is interesting to ask the question whether all the dSphs could be embedded in dark matter haloes of similar total mass. In Figure [5] the estimated mass to light ratios of the local group $\mathrm{dSphs}$ are plotted against their V-band absolute magnitudes. The figure is based on that given in Mateo et al. (1998) but includes more recent mass estimates where these are available. The solid curve shows the expected relation obtained for a population of objects whose stellar mass to light ratio is $1.5 \mathrm{M}_{\odot} / \mathrm{L}_{\odot}$ and whose total halo mass is $4 \times 10^{7} \mathrm{M}_{\odot}$. In this case, the total mass to light ratio is simply given by $(M / L)_{\text {tot }}=(M / L)_{\text {stars }}+M_{\mathrm{dm}} / L$, where $M_{\mathrm{dm}}$ is the mass in dark matter and $L$ is the total V-band luminosity. Although there is 


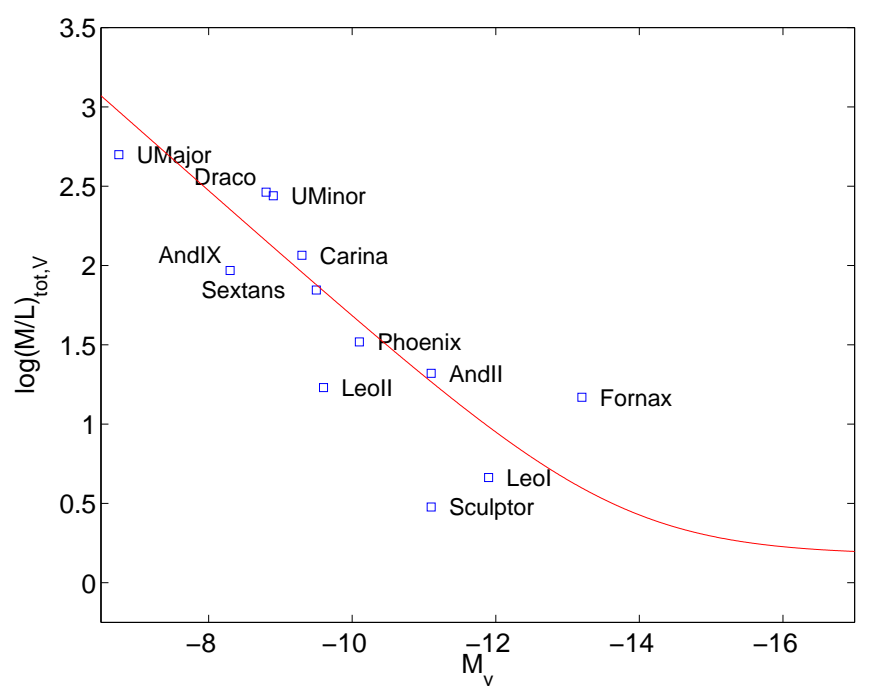

Fig. 5. Mass to light ratios versus absolute magnitudes for Local Group dwarf galaxies. Masses for Phoenix, LeoI, LeoII and Sculptor are taken from Mateo et al. (1998); the mass of AndII is taken from Côté et al. (1999). Recent mass estimates for the remaining $\mathrm{dSphs}$ are taken from the sources on these dSphs cited in the main text. See text for a discussion.

considerable scatter about this relation, it appears that most observations to date are consistent with dSphs having a common halo mass scale of around $4 \times 10^{7} \mathrm{M}_{\odot}$. One might legitimately ask whether this plot contains any more information than the similarity of dSph velocity dispersions. However, although all dSphs seem to have velocity dispersions of $6-10 \mathrm{~km} \mathrm{~s}^{-1}$, they have a wide range of spatial extents. For a tracer population in a fixed potential well, a larger spatial extent requires a larger velocity dispersion to support it. Thus, this plot demonstrates that it is possible to interpret the narrow range of $\mathrm{dSph}$ velocity dispersions in terms of a common mass scale. Given that the inclusion of radius information in the mass estimates is currently rather inhomogeneous, it will be interesting to see how more detailed mass modelling of the dSphs affects the details of this plot.

\section{Outlook}

As a result of the concerted efforts of a number of groups, high quality observed velocity dispersion profiles will soon be available for all the Milky Way dSphs. Improvements in the mass models are now required to take advantage of the richness of these data. Of particular interest are the correlations between kinematics and metallicity, recently highlighted by Tolstoy et al. (2004) for the case of Sculptor. In addition, the study of kinematic substructure in dSphs (e.g. Kleyna et al. 2003) has the potential to yield useful insights into the formation of dSphs. Other imminent observational developments include the extension of surveys to the M31 system (e.g. Chapman et al. 2005) and the search for even more dark matter 
dominated systems (e.g. the Ursa Major dSph: Willman et al. 2005; Kleyna et al. 2005).

\section{References}

Aaronson, M., 1983, ApJ, 266, L11

Aaronson, M., Olszewski, E., 1987, IAU Symp. 117, 153

Armandroff, T. E., Olszewski, E. W., Pryor, C., 1995, AJ, 110, 2131

Binney, J. J., Tremaine, S., 1987, Galactic Dynamics, Princeton University Press, Princeton

Chapman, S. C., Ibata, R., Lewis, G. F., Ferguson, A. M. N., Irwin, M., McConnachie, A., Tanvir, N., ApJL, in press, astro-ph/0506103

Côté, P., Mateo, M., Olszewski, E. W., Cook, K. H., 1999, ApJ, 526, 147

Klessen, R. S., Grebel, E. K., Harbeck, D., 2003, ApJ, 589, 798

Kleyna, J. T., Wilkinson, M. I., Evans, N. W., Gilmore, G., 2001, ApJ, 563, L115

Kleyna, J., Wilkinson, M. I., Evans, N. W., Gilmore, G., Frayn, C., 2002, MNRAS, 330, 792

Kleyna, J. T., Wilkinson, M. I., Gilmore, G., Evans, N. W., 2003, ApJ, 588, L21

Kleyna, J. T., Wilkinson, M. I., Evans, N. W., Gilmore, G., 2004, MNRAS, 354, L66

Kleyna, J. T., Wilkinson, M. I., Evans, N. W., Gilmore, G., 2005, ApJ, 630, L141

Koch, A., et al., 2005, AJ, submitted

Łokas, E. L., 2001, MNRAS, 327, L21

Łokas, E., Mamon, G., Prada, F., 2005, this proceedings

Majewski, S. R., et al., 2005, AJ, submitted, astro-ph/0503627

Mateo, M., 1997, ASP Conf. Ser. 116, 259

Mateo, M., 1998, ARA\&A, 36, 435

Mateo, M., Olszewski, E. W., Vogt, S. S., Keane, M. J., 1998, AJ, 116, 2315

Muñoz, R. R., et al., 2005, ApJ, astro-ph/0504035

Olszewski, E. W., Pryor, C., Armandroff, T. E., 1996, AJ, 111, 750

Press, W. H., Teukolsky, S. A., Vetterling, W. T., Flannery, B. P., 1992, Numerical Recipes in C. Cambridge Univ. Press, Cambridge

Pryor, C., Kormendy, J., 1990, AJ, 100, 127

Read, J. I., Wilkinson, M. I, Evans, N. W., Gilmore, G. F., Kleyna, J. T., 2005, MNRAS, submitted

Tolstoy, E., et al. 2004, ApJ, 617, L119

Wang, X., Woodroofe, M., Walker, M. G., Mateo, M., Olszewski, E., 2005, ApJ, 626, 145

Wilkinson, M. I., Kleyna, J. T., Evans, N. W., Gilmore, G. F., Irwin, M. J., Grebel, E. K., 2004, ApJ, 611, L21

Willman, B., et al., 2005, ApJ, 626, L85 